\title{
Analytic Methods for \\ the Modeling of Flexible Structures
}

\author{
J.M. Schumacher \\ Centre for Mathematics and Computer Science \\ P.O. Box 4079, 1009 AB Amsterdam. The Netherlands
}

\begin{abstract}
Standard approaches to the modeling of large flexible structures, such as the Finite Element Method, are essentially based on small-scale approximation: the structure is thought of as consisting of many pieces that are small enough to have their dynamics approximated by a low-order model. This modeling philosophy is suitable for complicated and inhomogeneous structures, but becomes awkward when one has to do with structures that are built up trom highly flexible but homogeneous parts. In the latter case, it would be much more natural to use partial differential equations. In this paper, we discuss some possible analytic approaches to the modeling of flexible structures, with an emphasis on the computation of the natural frequencies.
\end{abstract}

\section{INTRODUCTION}

Current developments in space technology tend towards the deployment of ever larger structures in space. As an example, very large solar panels will be needed to provide the energy for a space station. In spite of their extensiveness, such space structures will have to be light to help keep launching costs down, or to make launching feasible at all. As a result, engineers are facing a situation in which they have to deal with structures whose degree of flexibility is well beyond the limits that have been reached in earlier stages.

The dynamic modeling of such highly flexible structures will be a key step to take, and constitutes already a major challenge. Standard modeling tools such as the Finite Element Method are essentially based on the cutting up of a structure into many small pieces, which each receive their own low-order dynamic model. When a structure is very flexible, its dynamics can only be represented with reasonable accuracy in this way by considering a very large number of pieces, and this may make the modeling effort computationally infeasible. Therefore, it will be necessary to use every extra information that we have about a structure in order to bring down the computational load.

An example of such extra information is homogeneity. Consider the extreme case of a 'structure' consisting solely of a long, slender, and homogeneous beam. Using the Finite Element Method, one would consider the beam as a collection of many small parts, leading to a model of finite but high order from which one may, for instance, compute the natural frequencies. Alternatively, one can use the classical Euler-Bernoulli beam theory. Although this gives rise to an infinitedimensional model, the homogeneity of the beam guarantees that the solutions may be written down in terms of transcendental functions which can be expressed in various ways (e. g., as series expansions), so that it is also possible to calculate the natural frequencies and other properties from this representation. In fact, the finite element representation may be seen as one way of truncating the infinite-dimensional model given by the Euler-Bernoulli beam equation - not necessarily the most efficient way.

In this paper, we will discuss the modeling of structures that are built up by interconnecting a moderate number of 'distributed elements', each of which can be described by the classical equations of mathematical physics. Using the fact that these equations may be solved explicitly (i. e., the solutions can be expressed in terms of transcendental functions whose values may be determined to any desired accuracy), one obtains in this way a representation in terms of analytic matrices (matrices whose entries are analytic functions). This representation is more compact than the ones that would be obtained from the Finite Element Method or comparable techniques, but the compactness comes at a price: one will have to deal with parameter-dependent matrices, and these are not so easily manipulated as matrices whose entries are (real or complex) numbers.

In the next section, the construction of a distributed element representation of a flexible structure will be illustrated using some simple examples. Next, we will discuss techniques for operations on analytic matrices. These techniques may be applied to obtain methods for specific computational goals, and we shall discuss three such methods for the computation of the natural frequencies in Section 4. In the final section, we shall comment briefly on various other aspects of the modeling of flexible structures by analytic methods.

\section{THE SUBSTRUCTURE METHOD}

We want to consider models of a given physical structure that are obtained as connections of substructures. Therefore, the model will consist of a set of element descriptions together with a set of connection constraints. We start with the element descriptions.

In order to obtain a compact matrix representation, we note that it is sufficient to describe the behavior of the elements at the points where they are connected to eachother or to the 'outside world' (an active controller, perhaps). So the purpose is to write down a matrix which gives the dynamic relationships between generalized forces and generalized displacements at the connection points. How this can be done is best illustrated by a simple example. 
Perhaps the simplest distributed element is the vibrating string, whose equations are given as follows.

$$
\begin{aligned}
\mu \frac{\partial^{2}}{\partial t^{2}} w(x, t) & =T \frac{\partial^{2}}{\partial x^{2}} w(x, t) \\
-T \frac{\partial}{\partial x} w(0, t) & =F_{1}(t) \\
T \frac{\partial}{\partial x} w(l, t) & =F_{2}(t) \\
w(0, t) & =y_{1}(t) \\
w(l, t) & =y_{2}(t)
\end{aligned}
$$

Here, $w(x, t)$ is the displacement of the string (assumed in one direction only) at point $x$ at time $t, \mu$ is the mass density, $T$ is the tension, and $l$ is the length of the string. We assume that the extremes are taken as the points of connection; the forces and displacements at these points are given by $F_{1}(t), F_{2}(t), y_{1}(t)$, and $y_{2}(t)$. The general solution of $(2.1)$ for motion at frequency $\omega$ is given by the amplitude

$$
\phi(x)=a \cos \frac{\omega}{c} x+b \frac{c}{\omega} \sin \frac{\omega}{c} x
$$

where $c=(T / \mu)^{1 / 2}$ is the wave propagation speed. For $\omega=0$, the expression on the right hand side is to be read as $\phi(x)=a+b x$ (the analytic extension of $\phi$ when looked at as a function of $\omega$ ). The forces and displacements at the connection points can be expressed in terms of the parameters $a$ and $b$ :

$$
\begin{aligned}
& y_{1}(t)=\phi(0)=a \\
& y_{2}(t)=\phi(l)=a \cos \frac{\omega}{c} l+b \frac{c}{\omega} \sin \frac{\omega}{c} l \\
& F_{1}(t)=-\phi^{\prime}(0)=b T \\
& F_{2}(t)=\phi^{\prime}(l)=a T \frac{\omega}{c} \sin \frac{\omega}{c} l-b T \cos \frac{\omega}{c} l
\end{aligned}
$$

Elimination of the parameters $a$ and $b$ now leads to a description of the string in terms of matrices depending on $\omega$. In fact, there are several natural representations at hand: one may express the forces in terms of the displacements, which leads to the 'dynamic stiffness matrix' or 'impedance matrix'; conversely, one may express the displacements in terms of the forces, which leads to the 'dynamic elasticity matrix' or 'admittance matrix'. A third representation is obtained if one expresses the forces and displacements at the left hand side in terms of the forces and displacements at the right hand side, or vice versa; this way, one obtains what is sometimes called the 'transfer matrix' (for instance in [11]), or also (mostly in electrical engineering) the 'chain scattering matrix' or 'transmission matrix'. The transmission matrix is clearly useful when studying series connections of elements, but its use is not restricted to this domain (see [11] and also below). In our example, the transmission matrix (from the point ' $O$ ' to the point ' $P$ ) is

$$
T(\omega)=\left(\begin{array}{cc}
\cos \frac{\omega}{c} l & \frac{c}{\omega T} \sin \frac{\omega}{c} l \\
-T \frac{\omega}{c} \sin \frac{\omega}{c} l & -\cos \frac{\omega}{c} l
\end{array}\right)
$$

and we see that this is a matrix of analytic functions in $\omega$. In contrast, the impedance matrix is computed as

$$
Z(\omega)=\frac{-\sqrt{\mu T} \omega}{\sin \frac{\omega}{c} l}\left[\begin{array}{cc}
\cos \frac{\omega}{c} l & -1 \\
-1 & \cos \frac{\omega}{c} l
\end{array}\right]
$$

which is only a meromorphic matrix: it has poles as well as zeros. Note, however, that the impedance matrix appears originally (upon elimination of the parameters $a$ and $b$ from $(2.7-2.10)$ ) as a fraction of two analytic matrices:

$$
Z(\omega)=\left[\begin{array}{cc}
0 & T \\
T \frac{\omega}{c} \sin \frac{\omega}{c} l & -T \cos \frac{\omega}{c} l
\end{array}\right]\left[\begin{array}{cc}
1 & 0 \\
\cos \frac{\omega}{c} l & \frac{c}{\omega} \sin \frac{\omega}{c} l
\end{array}\right]^{-1} .
$$

This fractional form should be considered as the 'natural form' in which the impedance matrix of an element appears. Similar statements can be made for the admittance matrix.

In general, we shall assume that the (distributed) elements of a given structure are described by linear partial differential equations, whose solutions can be expressed in terms of well-defined transcendental functions (given, for instance, by a series development). Then, as above, one can write down expressions for generalized forces and generalized displacements at selected points on the element in terms of a number of free parameters ('integration constants'). This leads to a description of the element in a form which can generally be given as

$$
q_{\text {elt }}=Z_{q}^{\text {elt }}(\omega) \alpha_{\text {elt }}
$$




$$
Q_{e l t}=Z_{Q}^{e l t}(\omega) \alpha_{e l t}
$$

where $q_{e l t}$ is a vector of generalized displacements and $Q_{e t}$ is a vector of generalized forces.

We now have a description of the separate elements; we have to add connection constraints in order to combine the elements into a structure. We shall assume that the connection constraints may be given as linear relations between the displacements, so that the connections are represented by an equation

$$
C_{q}=0
$$

where $C$ is a constant matrix and $q$ is a vector of displacements, obtained by stacking the displacement vectors of the elements of the structure. It is natural to assume that the rows of the matrix $C$ are linearly independent; this just means that there are no redundant constraints. Then, if $q$ is an $n$-vector and $C$ is an $(n-k) \times n$-matrix, we can say that the number of degrees of freedom (in displacement) is $n$ before connection, and $k$ after connection.

The equations for the forces and displacements on the elements can be collected into two equations of the form

$$
\begin{aligned}
& q=Z_{q} \alpha \\
& Q=Z_{Q} \alpha
\end{aligned}
$$

where $Z_{q}$ and $Z_{Q}$ are block diagonal matrices, with on the blocks the $Z_{q}^{\text {elt }}$ - and $Z_{Q}^{\text {elt }}$-matrices of the elements. In order to arrive at a description of the connected structure, we now want to select $k$ displacement variables which correspond to the $k$ remaining degrees of freedom after connection. Also, rather than explicitly solving for the constraint forces due to the connection, we want to select a number of forces which may still be freely prescribed.

With the connection constraint on the displacement variables given by a matrix $C$ having independent rows, it is natural to introduce as 'free' variables a vector

$$
q_{1}=C_{1} q
$$

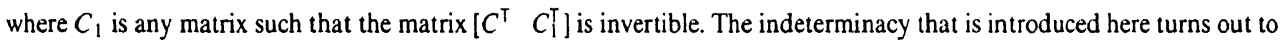
be irrelevant for the computation of the natural frequencies. In order to select new force variables, we appeal to the notion of energy. It will be assumed that force and displacement coordinates in the element descriptions have been chosen in such a way that the rate of change of energy for the unconnected structure is given by

$$
\dot{E}=\dot{q}^{\top} Q
$$

Now, write

$$
\left(\begin{array}{c}
C \\
C_{1}
\end{array}\right)^{-1}=\left(\begin{array}{c}
F \\
F_{1}
\end{array}\right)^{\top}
$$

We can compute the following expression for the rate of change of energy under the constraint (2.16):

$$
\dot{E}=\dot{q}^{\top} Q=\left(\left[\begin{array}{c}
F \\
F_{1}
\end{array}\right]^{\top}\left(\begin{array}{c}
C \\
C_{1}
\end{array}\right) \dot{q}\right)^{\top} Q=\left(F_{1}^{\top} \dot{q}_{1}\right)^{\top} Q=\dot{q}_{1}^{\top} F_{1} Q .
$$

So if we define

$$
Q_{1}=F_{1} Q,
$$

the rate of change of energy under the constraint (2.16) will be given by $\dot{E}=\dot{q}_{1}^{\top} Q_{1}$. As a description of the connected structure, we shall therefore take the dynamic equations (2.17-2.18), together with the connection constraint (2.16); the displacement variables will be given by (2.19) and the force variables by (2.23), while the matrices $C_{1}$ and $F_{1}$ are connected through (2.21).

The resonance frequencies of a flexible structure are defined as the frequencies at which the system can be in harmonic motion without any forces other than constraint forces acting. So, these are the frequencies $\omega_{0}$ for which there exists a vector $\alpha_{0}$ such that we will have

$$
C Z_{q}\left(\omega_{0}\right) \alpha_{0}=0
$$

as well as

$$
F_{1} Z_{Q}\left(\omega_{0}\right) \alpha_{0}=0
$$

The form of the equations here corresponds to harmonic motion; the first equation ensures that the connection constraints are satisfied, the second equation states that no forces are acting except constraint forces. In other terms, the resonance frequencies correspond to those values of $\omega$ for which the matrix

$$
\left(\begin{array}{c}
C Z_{q}(\omega) \\
F_{1} Z_{Q}(\omega)
\end{array}\right)
$$

has less than full column rank. Note that this matrix is square; its dimension is $n$ where $n$ is the the total number of degrees of freedom in displacement before connection (this may be in the order of a few hundred for a satellite). It is an analytic matrix, and, based on reasonable assumptions involving the positivity of the energy function (cf. [16]), it can be shown that its determinant is not identically zero. 
It may happen that, at certain frequencies $\omega_{0}$, there exist vectors $\alpha_{0}$ such that not only (2.24) and (2.25) are satisfied, but also

$$
C_{1} Z_{q}\left(\omega_{0}\right) \alpha_{0}=0
$$

Such frequencies will be called pure force frequencies, because it follows from (2.24) and (2.27) that the corresponding vibration mode has a zero displacement vector. Resonance frequencies that are not pure force frequencies will be called displacement frequencies. More precisely, the multiplicity of a displacement frequency $\omega_{0}$ is defined as the multiplicity of $\omega_{0}$ as a zero of the matrix (2.26) minus its multiplicity as a zero of the matrix

$$
\left(\begin{array}{c}
Z_{q}(\omega) \\
F_{1} Z_{Q}(\omega)
\end{array}\right)
$$

These definitions may be illustrated in a very simple finite-dimensional example. Consider two masses with one degree of freedom that are connected by a spring. Suppose that we want to model the mass of the spring as well; with proper normalization, we can do this by letting the two mass points at the ends have mass 1 , and by assuming that they are connected by two springs with spring constant 1 , coupled by a mass $m$. This leads to the following model:

$$
\begin{aligned}
& {\left[\begin{array}{l}
q_{1} \\
q_{2}
\end{array}\right]=\left[\begin{array}{cc}
1 & 0 \\
\left(\omega^{2}-1\right)\left(m \omega^{2}-2\right)-1 & m \omega^{2}-2
\end{array}\right]\left[\begin{array}{l}
\alpha_{1} \\
\alpha_{2}
\end{array}\right] .} \\
& \left.\left[\begin{array}{l}
Q_{1} \\
Q_{2}
\end{array}\right]=\left(\begin{array}{cc}
0 & 1 \\
-\omega^{2}\left(\omega^{2}-1\right)\left(m \omega^{2}-(m+2)\right) & 1-\left(\omega^{2}-1\right)\left(m \omega^{2}-2\right)
\end{array}\right]\right)\left[\begin{array}{l}
\alpha_{1} \\
\alpha_{2}
\end{array}\right] .
\end{aligned}
$$

Now, assume that the two end masses are constrained, by some mechanism, to move only in opposite directions. This constraint is expressed by the matrix

$$
C=\left[\begin{array}{ll}
1 & 1
\end{array}\right] \text {. }
$$

We may take $C_{1}=\left[\begin{array}{ll}1 & 0\end{array}\right]$, which corresponds to letting the variable $q_{1}$ play the role of 'free variable'. As a consequence, one obtains $F_{1}=\left[\begin{array}{ll}1 & -1\end{array}\right]$. The matrix (2.26) becomes

$$
\left[\begin{array}{cc}
\left(\omega^{2}-1\right)\left(m \omega^{2}-2\right) & m \omega^{2}-2 \\
\omega^{2}\left(\omega^{2}-1\right)\left(m \omega^{2}-(m+2)\right) & \left(\omega^{2}-1\right)\left(m \omega^{2}-2\right)
\end{array}\right] .
$$

This matrix has zeros at $\omega= \pm 1$ and at $\omega= \pm \sqrt{2 / m}$, so the displacements frequencies are at 1 and $\sqrt{2 / m}$ in normalized units. To find the pure force frequencies, we have to check whether the matrix

$$
\left[\begin{array}{cc}
1 & 0 \\
\left(\omega^{2}-1\right)\left(m \omega^{2}-2\right)-1 & m \omega^{2}-2 \\
-\omega^{2}\left(\omega^{2}-1\right)\left(m \omega^{2}-(m+2)\right) & \left(\omega^{2}-1\right)\left(m \omega^{2}-2\right)
\end{array}\right]
$$

has any zeros. One sees, in fact, that the above matrix does have zeros at $\pm \sqrt{2 / m}$, so the ' $\sqrt{2 / m}$ ' frequency is a pure force frequency. In the corresponding vibration mode, the middle mass moves in between the two outer masses; the end masses are not moving because there are forces acting on them that exactly counteract the forces that are due to the vibrations of the middle mass. These forces always point in the same direction, so they are not constraint forces. The example shows that pure force frequencies do occur; of course, it had to be specially prepared for this purpose - nonsquare matrices are not, in general, expected to have zeros.

The numerical determination of the natural frequencies is the first and foremost problem in the vibration analysis of flexible structures. The frequencies are given as the zeros of the matrix (2.26), which can be written down from the specification of the structure. The size of this matrix, however, is equal to the number of degrees before connection; even when distributed elements are being used, this may be in the order of a few hundred for a satellite with several appendages. Although this number is quite small in comparison with the sizes of matrices that would appear in a 'finite element' approach, we have to take in to account that we are dealing here with parameter-dependent matrices, and a matrix size of a few hundred has then to be considered as infeasibly large. So, an important problem that comes up here is: how to express the resonance frequencies as the zeros of a matrix that has a more manageable size. Below, we shall discuss three different methods to do this, but first we need some more material about the handling of analytic matrices.

\section{OPERATIONS ON ANALYTIC MaTRICES}

Let us first introduce some notation and terminology. We let $H$ (for holomorphic) denote the ring of functions that are analytic on the entire complex plane $\mathbf{C}$. The field of meromorphic functions on $\mathbf{C}$ (i.e., functions that are analytic everywhere except on a set having no finite limit point, where they have poles) will be denoted by $M$. It is a standard fact (see, for instance, $[4$, p. 173]) that $M$ is the quotient field of $H$. The set of matrices of size $p \times m$ with entries in $H$ (resp. $M$ ) will be denoted by $H^{p \times m}$ (resp. $M^{p \times m}$ ). An element of $H^{p \times p}$ is said to be unimodular if it has an inverse in $H^{p \times p}$. One can easily verify that an analytic matrix is unimodular if and only if it is invertible at every point of $C$.

The theory of analytic matrices is to a large extent analogous to the theory of polynomial matrices, which, by now, is a well-established tool in linear system theory, as is evidenced by the large number of textbooks in which polynomial matrices play a leading role: see $[1,2,8,9,14,20]$. There is a significant point of difference, though: because analytic functions may have an infinite number of zeros, the set $H$ does not form a factorization domain. This implies that $H$ is not a 
principal ideal domain, unlike the ring of polynomials $\mathbb{R}[s]$. However, $H$ does have the property that to every two elements $\phi$ and $\psi$ of $H$, not both equal to zero, there exists a greatest common divisor $\theta$, which can, moreover, always be written as a linear combination of $\phi$ and $\psi$ with coefficients in $H$ :

$$
\theta(z)=a(z) \phi(z)+b(z) \psi(z)
$$

where both $a(z)$ and $b(z)$ are elements of $H$. The existence of a greatest common divisor follows from a theorem of Weierstrass; the 'Bézout property' (3.1) can be derived from Mittag-Leffler's theorem. If one has to do with polynomials, the Euclidean algorithm can be used to find the 'Bézout factors' $a(z)$ and $b(z)$. In the analytic case, a different method has to be employed. Wedderburn [18] gives the following construction. First, one may assume that the greatest common divisor of $\phi(z)$ and $\psi(z)$ is 1 (otherwise, common factors are removed first). So, we are given two analytic functions $\phi(z)$ and $\psi(z)$ that do not have any zeros in common, and we are looking for analytic functions $a(z)$ and $b(z)$ such that

$$
a(z) \phi(z)+b(z) \psi(z)=1 .
$$

The meromorphic function $(\phi(z) \psi(z))^{-1}$ has poles at the zeros of $\phi(z)$ and at the zeros of $\psi(z)$. According to MittagLeffler's theorem, there exist meromorphic functions $c(z)$ and $d(z)$ whose poles and singular parts correspond exactly to those of $(\phi(z) \psi(z))^{-1}$ at the zeros of $\phi(z)$ and $\psi(z)$, respectively; so we can write

$$
(\phi(z) \psi(z))^{-1}=c(z)+d(z)+f(z)
$$

where $f$ is an analytic function. Moreover, the functions $c(z)$ and $d(z)$ can be constructed effectively - this may not be immediately clear from proofs of Mittag-Leffler's theorem in modern textbooks, but it is quite transparent in more classical treatments such as [7]. Now, take

$$
a(z)=\psi(z)(d(z)+f(z)), \quad b(z)=\phi(z) c(z) ;
$$

then both $a(z)$ and $b(z)$ are analytic, and they satisfy the desired equality.

This result may also be stated as follows: an arbitrary nonzero $2 \times 1$-matrix of analytic functions can be reduced to a form in which the bottom entry is zero by premultiplication by a unimodular matrix. Indeed, if $\phi(z)$ and $\psi(z)$ are analytic functions (not both equal to zero) and $\theta(z)$ is their greatest common divisor, then we have, writing $\phi_{1}(z)=\phi(z) \theta^{-1}(z)$ and $\psi_{1}(z)=\psi(z) \theta^{-1}(z)$,

$$
\left[\begin{array}{cc}
a(z) & b(z) \\
-\psi_{1}(z) & \phi_{1}(z)
\end{array}\right]\left[\begin{array}{l}
\phi(z) \\
\psi(z)
\end{array}\right)=\left(\begin{array}{c}
\theta(z) \\
0
\end{array}\right)
$$

where the $2 \times 2$-matrix on the left is unimodular. Moreover, it can easily be verified that every $\theta(z)$ that appears in a multiplication of this type must be a greatest common divisor of $\phi(z)$ and $\psi(z)$. The construction is easily extended to the case of a $p \times 1$-matrix: repeated applications of the above, with row interchanges if necessary, can be used to show that such an analytic matrix may be reduced, by premultiplication by a unimodular matrix, to a form in which only the top entry is non-zero. Repeated application of this routine, in its turn, will reduce a general $p \times m$ analytic matrix to upper triangular form (up to column permutations; it is easily seen, however, that no column permutations will be necessary when the given matrix has full normal column rank). Of course, the technique is the one that is generally used to reduce a matrix over a Bézout domain to upper triangular form (cf., for instance, [10, p. 32]). The result is most often applied in the following form.

Proposition 3.1 For any matrix $T(z)$ over $H$, one can find (constructively, in principle) a unimodular matrix $U(z)$ over $H$ such that

$$
U(z) T(z)=\left[\begin{array}{l}
R(z) \\
0
\end{array}\right.
$$

where $R(z)$ has full row rank (as a matrix over the field $M$ ).

Many of the well-known techniques of polynomial matrix theory are based on the analogous result for polynomial matrices, and so we can extend these techniques to the domain of analytic matrices. As an example, consider the problem of $G C D$ extraction, which comes up in many applications including coprime factorization.

The coprime factorized form for matrices in $M^{p \times m}$ is defined as follows. Two matrices $N \in H^{p \times m}$ and $D \in H^{k \times m}$ are said to be right coprime if the stacked matrix $\left[N^{\top} D^{\top}\right]^{\top}$ has full column rank everywhere on $\mathrm{C}$ (equivalently, this matrix has a left inverse in $H^{m \times(p+k)}$. Suppose now that $D$ is square and of full normal rank; then $P$ defined by $P(z)=N(z) D^{-1}(z)$ is a meromorphic matrix, which is said to appear in right coprime factorized form. The left coprime factorized form is defined analogously.

Suppose now that a meromorphic matrix appears in a factorized form $P(z)=N(z) D^{-1}(z)$, but the factors $N(z)$ and $D(z)$ are not right coprime. Then the reduction to upper triangular form that was described above can be used to extract a greatest common divisor from $N(z)$ and $D(z)$, as follows. Let $U$ be a unimodular matrix such that

$$
U\left(\begin{array}{l}
D \\
N
\end{array}\right)=\left(\begin{array}{l}
T \\
0
\end{array}\right) .
$$

The matrix $T(z)$ must be square and invertible. Let $U^{-1}=V$, so that

$$
\left(\begin{array}{l}
D \\
N
\end{array}\right)=\left(\begin{array}{ll}
V_{11} & V_{12} \\
V_{21} & V_{22}
\end{array}\right)\left(\begin{array}{l}
T \\
0
\end{array}\right)
$$


We then have $D=V_{11} T$ and $N=V_{21} T$, so that a right coprime factorization of $P(z)$ is now given by $P(z)=V_{21}(z) V_{11}^{-1}(z)$.

The same technique applied to coprime factorizations leads to a matrix analogon of (3.2). Let $P(z)=N(z) D^{-1}(z)$ be a right coprime factorization; then there exists a unimodular matrix $U(z)$ such that

$$
\left(\begin{array}{ll}
U_{11} & U_{12} \\
U_{21} & U_{22}
\end{array}\right]\left[\begin{array}{c}
D \\
N \\
-
\end{array}\right)=\left(\begin{array}{l}
R \\
0
\end{array}\right)
$$

Here, the matrix $R$ is square and has full rank for all $z \in \mathrm{C}$, so that $R$ is, in fact, unimodular. From the above, we immediately get

$$
A(z) D(z)+B(z) N(z)=I
$$

if we take $A(z)=R^{-1}(z) U_{11}(z)$ and $B(z)=R^{-1}(z) U_{12}(z)$.

In local questions, there is no essential difference between meromorphic matrices and rational matrices, unless, of course, one would like to discuss the behavior at infinity. At the finite points of the complex plane, one may, for instance, very well define the poles and zeros of a meromorphic matrix by using the ring $H_{\alpha}$ of functions that are analytic in a neighborhood of $\alpha$. This is done in the following way. Let $P(z)$ be a meromorphic matrix. Write its Laurent series development around $\alpha$ :

$$
P(z)=P_{-k}(z-\alpha)^{-k}+\cdots+P_{-1}(z-\alpha)^{-1}+P_{0}+P_{1}(z-\alpha)+\cdots .
$$

It is clear that $(z-\alpha)^{k} P(z)$ is a matrix over $H_{\alpha}$. Now, $H_{\alpha}$ is a principal ideal domain and so we can write a 'local Smith form' for $(z-\alpha)^{k} P(z)$ at $\alpha([10$, p. 43]):

$$
(z-\alpha)^{k} P(z)=U(z)\left(\begin{array}{cc}
\Delta(z) & 0 \\
0 & 0
\end{array}\right) V(z)
$$

where both $U(z)$ and $V(z)$ are unimodular over $H_{\alpha}$, and

$$
\Delta(z)=\operatorname{diag}\left((z-\alpha)^{\nu_{1}}, \cdots,(z-\alpha)^{\prime \prime}\right) .
$$

This immediately leads to a 'local Smith-McMillan form', given by

$$
P(z)=U(z)\left(\begin{array}{cc}
D(z) & 0 \\
0 & 0
\end{array}\right) V(z)
$$

where

$$
D(z)=\operatorname{diag}\left((z-\alpha)^{\kappa_{1}}, \cdots,(z-\alpha)^{\alpha_{\prime}}\right) .
$$

It can be shown, by standard methods, that the integers $\kappa_{1}, \cdots, \kappa_{r}$ that appear in this decomposition are determined uniquely by $P(z)$ and $\alpha$. We may assume that the $\kappa_{i}$ 's are ordered, so that

$$
\kappa_{1} \leqslant \cdots \leqslant \kappa_{r_{1}}<0=\kappa_{r_{1}+1}=\cdots=\kappa_{r_{2}}<\kappa_{r_{2}+1} \leqslant \cdots \leqslant \kappa_{r_{2}+r_{3}} .
$$

We then say that $P(z)$ has $r_{1}$ poles of orders $-\kappa_{1}, \cdots,-\kappa_{r_{1}}$ at $\alpha$, and that it has $r_{3}$ zeros of orders $\kappa_{r_{2}+1}, \cdots, \kappa_{r_{2}}+r_{3}$ at $\alpha$. In particular, the total zero multiplicity of $P(z)$ at $\alpha$, denoted by $z_{\alpha}(P)$, is

$$
z_{\alpha}(P)=\sum_{i=1}^{r_{3}} \kappa_{r_{2}+i} .
$$

\section{THREE COMPRESSION METHODS}

In this section, we shall discuss three methods to obtain a matrix of reasonably small size from which the frequencies (or more in particular, the displacement frequencies) of a structure can be determined. There are many factors that play a role in the assessment of the value of such methods: ease of implementation, computational demands, numerical reliability, scope of applicability, to name a few. We shall add comments on some of these aspects for each method.

Compression to a meromorphic matrix

The first method is based on the observation that one can write

$$
\left(\begin{array}{cc}
F_{1} Z_{Q} Z_{q}^{-1} F_{1} & F_{1} Z_{Q} Z_{q}^{-1} F^{\top} \\
0 & I
\end{array}\right)=\left(\begin{array}{c}
F_{1} Z_{Q} \\
C Z_{q}
\end{array}\right)\left(\left(\begin{array}{c}
C_{1} \\
C
\end{array}\right) Z_{q}\right)^{-1} .
$$

The matrix (2.26) appears here as the numerator matrix in a factorization of a meromorphic matrix that has a particularly simple form: note that the matrix $F_{1} Z_{Q} Z_{q}^{-1} F_{\mathrm{T}}^{\mathrm{T}}$ appearing in the upper left corner has size $k \times k$, where $k$ is the number of degrees of freedom after connection. One has to take into account the fact that the factorization appearing in (4.1) is not necessarily coprime. However, we have the following proposition, which shows that possible cancellations involve exactly the 'pure force frequencies'. In order to maximize confusion, we shall follow the notational conventions of system theory below and use ' $s$ ' as a complex variable, after we have already used the frequency parameter ' $\omega$ ' of dynamic analysis and the variable ' $z$ ' of function theory. 
Proposition 4.1 Let $N(s)$ and $D(s)$ be analytic matrices; suppose that $N(s)$ is injective, and that $D(s)$ is invertible. Under these conditions, the total zero multiplicities of $N(s),\left[N(s)^{\top} D(s)^{\top}\right]^{\top}$, and $N(s) D^{-1}(s)$ at a given point a of the complex plane are related as follows:

$$
z_{a}\left(N D^{-1}\right)=z_{\alpha}(N)-z_{\alpha}\left(\left[\begin{array}{l}
N \\
D
\end{array}\right)\right)
$$

Proof (Sketch) For any meromorphic matrix $G(s)$ of size $p \times m$ and for any given $\alpha \in \mathbb{C}$, define $\phi_{\alpha}(G)$ as the mapping from $H^{m} / H_{\alpha}^{m}$ to $H^{p} /\left(H_{\alpha}^{p}+G(s) H_{\alpha}^{m}\right)$ that is induced by $G(s)$ :

$$
\phi_{\alpha}(G): f(s)+H_{\alpha}^{m} \mapsto G(s) f(s)+\left(H_{\alpha}^{p}+G(s) H_{\alpha}^{m}\right) .
$$

This mapping is easily seen to be well-defined. If $G(s)$ is injective, one has (see [3]):

$$
z_{\alpha}(G)=\operatorname{dim} \operatorname{ker} \phi_{\alpha}(G) .
$$

Now, in the situation stated in the proposition, one verifies by computation that

$$
\phi_{\alpha}\left(D^{-1}\right)\left[\operatorname{ker} \phi_{\alpha}\left(N D^{-1}\right)\right]=\pi\left[\operatorname{ker} \phi_{\alpha}(N)\right]
$$

where $\pi$ is the natural projection from $H^{m} / H_{\alpha}^{m}$ to $H^{m} /\left(H_{\alpha}^{m}+D^{-1} H_{\alpha}^{m}\right)\left(=H^{m} / D^{-1} H_{\alpha}^{m}\right)$, and, of course, $m$ is the size of $D(s)$. Since $\phi_{\alpha}\left(D^{-1}\right)$ is injective, (4.5) gives

$$
\operatorname{dim} \operatorname{ker} \phi_{\alpha}\left(N D^{-1}\right)=\operatorname{dim} \operatorname{ker} \phi_{\alpha}(N)-\operatorname{dim}\left(\operatorname{ker} \phi_{\alpha}(N) \cap \operatorname{ker} \pi\right) .
$$

It is readily verified that $\operatorname{ker} \pi=\operatorname{ker} \phi_{\alpha} D$, and that

$$
\operatorname{ker} \phi_{\alpha}(N) \cap \operatorname{ker} \phi_{\alpha}(D)=\operatorname{ker} \phi_{\alpha}\left(\left[\begin{array}{l}
N \\
D
\end{array}\right)\right)
$$

Together with (4.4), this gives the desired result.

The method can be presented in a particularly simple way by letting $F_{1}$ obtain a special form. By a suitable change of coordinates in the vector space of displacements, it is possible to write the connection constraint matrix in the form $C=\left[\begin{array}{ll}0 & I_{n-k}\end{array}\right]$. This can be effected by a standard numerical procedure based on the singular value decomposition. Then one can, obviously, take $C_{1}=\left[\begin{array}{ll}I_{k} & 0\end{array}\right]$, which leads to an $F_{1}$-matrix which also has the form $\left[\begin{array}{ll}I_{k} & 0\end{array}\right]$. Now, consider the displacement-to-force transfer:

$$
Q=Z_{Q} Z_{q}^{-1} q \text {. }
$$

In the new coordinates, we can write this as

$$
\left(\begin{array}{l}
Q_{1} \\
Q_{2}
\end{array}\right)=\left(\begin{array}{ll}
Z_{11} & Z_{21} \\
Z_{21} & Z_{22}
\end{array}\right)\left[\begin{array}{l}
q_{1} \\
q_{2}
\end{array}\right)
$$

where $q_{1}$ and $Q_{1}$ are the retained displacements and forces, respectively. The connection constraint is given by $q_{2}=0$, and so the transfer between the retained forces and displacements is clearly

$$
Q_{1}=Z_{11} q_{1} \text {. }
$$

Moreover, the matrix $Z_{11}$ is, in these coordinates, exactly equal to $F_{1} Z_{Q} Z_{g}^{-1} F_{1}^{\top}$.

This compression method is well-tested. The method is used in the DISTEL program [12] developed by D. Poelaert at ESTEC for the dynamic analysis of flexible spacecraft. Unlike $Z_{4}$ and $Z_{Q}$, the matrix $Z_{11}$ contains poles as well as zeros; however, for the case of undamped, non-gyroscopic structures, $Z_{11}$ is a symmetric matrix all of whose zeros are located on the real line (corresponding to the imaginary axis in the $s$-plane of the Laplace transformation), which means that powerful numerical techniques such as the Wittrick-Williams method [19] can be used to find the zeros.

There are some precautions that have to be taken in relation to the possibility of pole-zero cancellation, which is inherently present when one is working with meromorphic matrices. A simple example of cancellation in the determinant is provided by the string, for which the impedance matrix (displacement-to-force transfer ratio) was given in (2.12). The determinant of this matrix is determined as

$$
\operatorname{det} Z(\omega)=-\mu T \omega^{2}
$$

which only has a (double) zero at 0 ; but this is not to say, of course, that the string does not have any non-rigid vibration modes. One should also be careful not to assume that the zeros of the matrix at the left hand side of (4.1) are given solely by the zeros of $F_{1} Z_{Q} Z_{q}^{-1} F_{\mathrm{I}}^{\mathrm{T}}$; there may also be zeros at the poles of $F_{1} Z_{Q} Z_{q}^{-1} F^{\top}$. It is seen that these poles can occur only at the zeros of $Z_{q}$, the so-called 'clamped frequencies'; these frequencies can relatively easily be calculated beforehand, since they depend only on the elements and not on the connection.

The DISTEL program has been successfully applied for the determination of the natural frequencies and vibration modes of a variety of ( structures, including the SCOLE experiment [13]. It takes full advantage of the special structure of non-gyroscopic, undamped mechanical structures. If one is interested in larger classes of systems (allowing for damping, gyroscopic effects, presence of non-mechanical elements such as active controllers), it becomes worthwile to look at methods based on other forms of compression. 
The transmission matrix

The second scheme we shall consider is based on the 'chain structure' of the object under study and, in fact, its success is dependent on the degree to which the structure under consideration is chain-like. To see how the 'chain structure' can be exploited, take another look at the factors in the representation (2.13) for the string impedance. We can split the forces and displacements into those that are associated with the 'left' side of the string and those that are associated with the 'right' side of the string. With proper scaling, one can write

$$
\left(\begin{array}{l}
Q_{r} \\
Q_{l}
\end{array}\right)=\left(\begin{array}{cc}
T_{1}(s) & T_{2}(s) \\
1 & 0
\end{array}\right]\left[\begin{array}{l}
a \\
b
\end{array}\right],\left(\begin{array}{l}
q_{r} \\
q_{l}
\end{array}\right)=\left(\begin{array}{cc}
T_{3}(s) & T_{4}(s) \\
0 & 1
\end{array}\right]\left(\begin{array}{l}
a \\
b
\end{array}\right)
$$

where the $T_{i}(s)(i=1, \cdots, 4)$ are analytic functions. Clearly, we might also specify the dynamics of the system by the equation

$$
\left(\begin{array}{l}
Q_{r} \\
q_{r}
\end{array}\right)=\left(\begin{array}{ll}
T_{1} & T_{2} \\
T_{3} & T_{4}
\end{array}\right)\left(\begin{array}{l}
Q_{l} \\
q_{l}
\end{array}\right) .
$$

The matrix that appears here is the 'transmission matrix'. We see that it is an analytic matrix. This is explained physically because the presence of a pole in this matrix would imply that there could be a nonzero harmonic motion of forces and/or displacements at the right hand side of the string while forces and displacements at the left end of the string would be zero. By the connectedness of the string, this is impossible. In fact, the same argument with 'left' and 'right' interchanged leads one to expect that $T^{-1}(s)$ will also be analytic, and this is borne out by computation. So, the matrix $T(s)$ is, in fact, unimodular. Another interesting feature of the representation (4.13) is that it corresponds to a right coprime factorization as given (implicitly) by (4.12), but, just as easily, also to a left coprime factorization:

$$
\left[\begin{array}{cc}
0 & T_{2} \\
I & -T_{4}
\end{array}\right]\left(\begin{array}{l}
q_{r} \\
q_{l}
\end{array}\right)=\left(\begin{array}{cc}
I & -T_{1} \\
0 & T_{3}
\end{array}\right)\left(\begin{array}{l}
Q_{r} \\
Q_{l}
\end{array}\right) .
$$

The 'connectedness' property described above for the string is not unique to that element; based on physical reasoning, one would expect that for many elements it should be possible to divide the (generalized) force and displacement coordinates in two groups in such a way that the vanishing of the coordinates in one group implies the vanishing of those in the other group as well. By a suitable choice of coordinate bases, one should then be able to obtain factors for the element in the form

$$
Z_{Q}=\left(\begin{array}{cc}
T_{1} & T_{2} \\
I & 0
\end{array}\right), \quad Z_{q}=\left(\begin{array}{cc}
T_{3} & T_{4} \\
0 & I
\end{array}\right) .
$$

This particular form of the factors makes it possible to obtain a certain compression of matrices that are used to describe a connected structure; the degree of compression depends on the nature of the connection. The procedure is best illustrated in a few simple examples.

Let us first consider two elements in a series connection. The two elements are described by

$$
\left[\begin{array}{l}
Q_{r}^{i} \\
Q_{i}^{i}
\end{array}\right]=\left(\begin{array}{cc}
T_{1}(s) & T_{2}^{i}(s) \\
1 & 0
\end{array}\right]\left[\begin{array}{l}
a \\
b
\end{array}\right),\left(\begin{array}{l}
q_{r}^{i} \\
q_{i}^{i}
\end{array}\right]=\left(\begin{array}{cc}
T_{3}(s) & T_{4}^{i}(s) \\
0 & 1
\end{array}\right]\left[\begin{array}{l}
a \\
b
\end{array}\right)
$$

for $i=1,2$. The connection constraint for a series connection is given by

$$
\left[\begin{array}{llll}
0 & I & -I & 0
\end{array}\right]\left[\begin{array}{l}
q_{r}^{1} \\
q ! \\
q_{r}^{2} \\
q ?
\end{array}\right)=0,
$$

so the matrix $C$ is given by

$$
C=\left[\begin{array}{llll}
0 & I & -I & 0
\end{array}\right] \text {. }
$$

For $F_{1}$, we can take

$$
F_{1}=\left(\begin{array}{llll}
I & 0 & 0 & 0 \\
0 & I & I & 0 \\
0 & 0 & 0 & I
\end{array}\right)
$$

This leads to

$$
\left[\begin{array}{c}
C Z_{q} \\
F_{1} Z_{Q}
\end{array}\right)=\left(\begin{array}{cccc}
0 & I & -T_{3}^{2} & -T_{4}^{2} \\
T 1 & T_{2}^{1} & 0 & 0 \\
I & 0 & T_{1}^{2} & T_{2}^{1} \\
0 & 0 & I & 0
\end{array}\right) .
$$

This matrix has considerable structure, and we can compress it by using 'Gaussian elimination' steps. There are no 
questions of numerical accuracy here, since the manipulation is completely formal. Using the symbol ' $\sim$ ' for 'has the same zeros as', we can write

$$
\begin{aligned}
& \left.\qquad \begin{array}{cccc}
0 & I & -T_{3}^{2} & -T_{4}^{2} \\
T_{1}^{1} & T_{2}^{1} & 0 & 0 \\
I & 0 & T_{1}^{2} & T_{2}^{1} \\
0 & 0 & I & 0
\end{array}\right] \sim\left(\begin{array}{ccc}
0 & I & -T_{4}^{2} \\
T \mid & T_{2}^{1} & 0 \\
I & 0 & T_{2}^{2}
\end{array}\right] \sim\left(\begin{array}{cc}
I & -T_{4}^{2} \\
T_{2}^{1} & -T_{1}^{2} T_{2}^{2}
\end{array}\right) \sim \\
& \sim-T \mid T_{2}^{2}+T_{2}^{1} T_{4}^{2} .
\end{aligned}
$$

So, the original matrix of size $4 \times 4$ (measured in blocks) is now reduced to a matrix consisting of a single block. Next, we consider a parallel connection of two elements. Using the same element representations as above, we have this time

$$
C=\left(\begin{array}{cccc}
I & 0 & -I & 0 \\
0 & I & 0 & -I
\end{array}\right)
$$

so that one may take

$$
F_{1}=\left(\begin{array}{llll}
I & 0 & I & 0 \\
0 & I & 0 & I
\end{array}\right) .
$$

Following the same procedure of 'Gaussian elimination' as above, we obtain for this case:

$$
\begin{aligned}
\left(\begin{array}{c}
C Z_{q} \\
F_{1} Z_{Q}
\end{array}\right) & =\left(\begin{array}{cccc}
T_{1}^{1} & T_{2}^{1} & -T_{1}^{2} & -T_{2}^{2} \\
I & 0 & -I & 0 \\
T_{3}^{1} & T_{4}^{1} & T_{3}^{2} & T_{4}^{2} \\
0 & I & 0 & I
\end{array}\right) \sim \\
& \sim\left(\begin{array}{ccc}
T \mid & T_{1}^{1}+T_{2}^{2} & -T_{1}^{2} \\
I & 0 & -I \\
T_{3}^{1} & T_{4}^{1}-T_{4}^{2} & T_{3}^{2}
\end{array}\right) \sim\left(\begin{array}{cc}
T_{1}^{1}-T_{1}^{2} & T_{2}^{1}+T_{2}^{2} \\
T_{3}^{1}+T_{3}^{2} & T_{4}^{1}-T_{4}^{2}
\end{array}\right) .
\end{aligned}
$$

Here, the compression leads to a matrix of size $2 \times 2$ in blocks. Of course, the expression that we found for the case of a series connection could also have been found by directly multiplying the transmission matrices - in fact, this is the most obvious use of the transmission matrix representation, which also finds application in, for instance, optics. However, the example featuring the parallel connection shows that the representation can be put to good use in other circumstances as well.

We will not attempt here to derive an expression for the minimal size of the matrix that can be obtained through operations of 'Gaussian elimination' as shown above, although it may be reasonable to conjecture that the minimal achievable size, measured in blocks, equals the number of loops in the structure plus one. Our conclusion here is just that, depending on the type of structure one has at hand, it is possible to use transfer matrix techniques to arrive at a representation of the frequencies of the given structure by an analytic matrix whose size is a modest multiple of $k$, the number of degrees of freedom after connection. Transfer matrix methods are discussed in [11] and, more specifically with an eye on applications to space structures, in [5]; the interpretation that we gave here in terms of 'Gaussian elimination' seems to be new, however.

\section{An analytic matrix method}

It is possible to obtain a characteristic matrix for the frequencies of a connected structure which is analytic and which has size $k$, where $k$ is the number of degrees of freedom after connection. However, this does not come for free: quite a bit of formal manipulation will have to be involved. To show how this characteristic matrix can be obtained, we first need some preparatory material. We shall write $N$ rather than $Z_{q}$ and $D$ rather than $Z_{Q}$, in accordance with conventions in system theory.

Leмma 4.2 If $\tilde{D}(s)$ and $\tilde{N}(s)$ are left coprime analytic matrices such that $N D^{-1}=\tilde{D}^{-1} \tilde{N}$, then the frequencies of the connected structure can also be found as the zeros of the matrix

$$
\left(\begin{array}{cc}
\tilde{D}(s) & -\tilde{N}(s) \\
C & 0 \\
0 & F_{1}
\end{array}\right) .
$$

The exceptional frequencies can also be obtained as the zeros of the matrix

$$
\left(\begin{array}{c}
-\tilde{N}(s) \\
F_{1}
\end{array}\right) .
$$


Proof We again use the notation ' $\sim$ ' for 'has the same zeros as'. Let $X, Y, \tilde{X}$ and $\tilde{Y}$ be analytic matrices such that (cf. [8], p. 382)

$$
\left(\begin{array}{cc}
\tilde{D} & -\tilde{N} \\
X & Y
\end{array}\right)\left(\begin{array}{cc}
\tilde{X} & N \\
-\tilde{Y} & D
\end{array}\right)=I
$$

where both factors are square (and hence, by the above relation, unimodular). Then we can write

$$
\left(\begin{array}{cc}
\tilde{D}(s) & -\tilde{N}(s) \\
C & 0 \\
0 & F_{1}
\end{array}\right) \sim\left(\begin{array}{cc}
\tilde{D} & -\tilde{N} \\
C & 0 \\
0 & F_{1}
\end{array}\right)\left(\begin{array}{cc}
\tilde{X} & N \\
-\tilde{Y} & D
\end{array}\right) \sim\left(\begin{array}{cc}
I & 0 \\
C \tilde{X} & C N \\
-F_{1} \tilde{Y} & F_{1} D
\end{array}\right) \sim\left(\begin{array}{c}
C N \\
F_{1} D
\end{array}\right)
$$

which proves the first part of the statement. Also, we have

$$
\left(\begin{array}{c}
-\tilde{N} \\
F_{1}
\end{array}\right) \sim\left(\begin{array}{cc}
\tilde{D} & -\tilde{N} \\
I & 0 \\
0 & F_{1}
\end{array}\right]\left(\begin{array}{cc}
\tilde{X} & N \\
-\tilde{Y} & D
\end{array}\right) \sim\left(\begin{array}{cc}
I & 0 \\
X & N \\
-F_{1} \tilde{Y} & F_{1} D
\end{array}\right) \sim\left(\begin{array}{c}
N \\
F_{1} D
\end{array}\right) .
$$

This establishes the second part.

We can now prove:

Proposition 4.3 Suppose that $N D^{-1}$ is symmetric. If $U(s)=\left[\begin{array}{ll}U_{1}(s) & U_{2}(s)\end{array}\right]$ is any unimodular matrix such that

$$
C N(s)\left[U_{1}(s) \quad U_{2}(s)\right]=[T(s) 0]
$$

where $T(s)$ is nonsingular, then the displacement frequencies of the connected structure are given by the zeros of $F_{1} D(s) U_{2}(s)$.

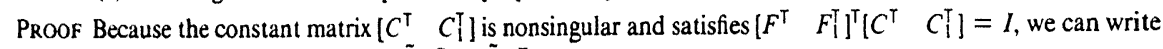

$$
\left[\begin{array}{l}
\tilde{N} \\
F_{1}
\end{array}\right) \sim\left(\begin{array}{c}
\tilde{N} \\
F_{1}
\end{array}\right)\left(\begin{array}{c}
C \\
C_{1}
\end{array}\right)^{\top}=\left(\begin{array}{cc}
\tilde{N} C^{\top} & \tilde{N} C_{1}^{\top} \\
0 & I
\end{array}\right) \sim \tilde{N} C^{\top} .
$$

From the symmetry assumption, it follows that $\tilde{N}^{\top}\left(\tilde{D}_{\tilde{D}}^{\top}\right)^{-1}$ is a right coprime factorization of $N D^{-1}$, so that there must exist a unimodular matrix $V$ such that $\tilde{N}^{\top}=N V$ and $\tilde{D}^{\top}=D V$. Since

$$
\left[\begin{array}{c}
C N(s) \\
F_{1} D(s)
\end{array}\right] \sim\left[\begin{array}{cc}
C N(s) U_{1}(s) & 0 \\
F_{1} D(s) U_{1}(s) & F_{1} D(s) U_{2}(s)
\end{array}\right]=\left[\begin{array}{cc}
T(s) & 0 \\
F_{1} D(s) U_{1}(s) & F_{1} D(s) U_{2}(s)
\end{array}\right)
$$

it only remains to prove that $T(s)$ has the same zeros as $\left[\tilde{N}^{\top}(s) F_{l}^{\top}\right]^{\top}$. This follows from:

$$
\tilde{N} C^{\top}=V^{\top} N^{\top} C^{\top} \sim N^{\top} C^{\top} \sim C N \sim\left[\begin{array}{ll}
T & 0
\end{array}\right] \sim T .
$$

It should be noted that equation (4.32) holds whether or not $N D^{-1}$ is symmetric. So the frequencies of the connected structure are always given by $F_{1} D(s) U_{2}(s)$, with omission of the zeros of $T(s)$.

The main difficulty in the approach suggested by the above result lies in the construction of the unimodular matrix $U(s)$. This involves the solution of many equations of the type

$$
a(s) \phi(s)+b(s) \psi(s)=1
$$

where $\phi(s)$ and $\psi(s)$ are given functions that have no zeros in common, and $a(s)$ and $b(s)$ are analytic functions that have to be constructed. It is possible 'in principle' to find $a(s)$ and $b(s)$ by a constructive procedure, as described Section 2; however, it remains to be seen how this might be implemented in software - it is certainly not a standard job for existing formal manipulation packages. So, while it may be attractive to have an analytic matrix of size $k \times k$ from which the frequencies of the connected structure may be computed, this must be weighed against the effort spent to arrive at such a matrix.

\section{Conclusions}

Parameter matrix modeling of flexible structures is a technique that has already shown its utility in the computation of the resonance frequencies of large structures. It is also a technique that holds promise for application to a larger domain which includes frequency computations for damped models, approximation, and controller design. However, this extension of the range of applications will require that a larger library of subroutines becomes available for the manipulation of parameter-dependent matrices. Among the facilities that one would in principle like to have available, we mention:

solution of the Bézout equation in matrices;

$\square$ additive decomposition of a meromorphic matrix in parts that have poles in designated regions;

- extraction of a greatest common divisor;

$\square$ coprime factorization in analytic matrices, or, more generally, in matrices that have poles in a designated region of the complex plane;

$\square$ inner/outer factorization. 
These are among the operations that are used, for instance in $[2,6,8,17]$, to solve a variety of modeling and control problems. The cited references also contain algorithms to perform these operations; however, all algorithms are restricted to the case of rational matrices and the extension to the case of meromorphic matrices will, in general, be nontrivial. It should be noted that the operations described above are not independent - some of them are used in others. By analysis, one can identify a limited number of 'basic routines' which form the elementary building blocks for all operations.

In this note, the example of computation of resonance frequencies has been chosen in order to illustrate some of the points that come up in the manipulation of parameter-dependent matrices. It is seen that there is a relation, which may take the form of a trade-off, between the procedures for formal manipulation that one is willing to use and the numerical procedures that are used to calculate the final results. One of the factors that have to be taken into account consists of the specific properties of the system under consideration: damped or undamped, gyroscopic or non-gyroscopic. Of course, there will also be an influence of the compututional gouls one has, which may range from determination of resonance frequencies and the corresponding modes to passive or active controller design.

Generally, one may say that the modern control theory literature tends to make liberal use of the availability of various highly structured representations for linear dynamical systems, such as the state space form and the coprime factorized form. This is certainly justifiable from the theoretical point of view, because one can prove that, under very general circumstances, such representations can always be found - at least in principle. However, in applications where models are built up as connections of subsystems, one has to start with system descriptions that are less well structured. In such cases, there will be a gap between the given form of the system and the form that is required to apply the theory, and one will have to bridge this gap either by re-writing the system equations in a suitable way, or by reformulating the theory in such a way that it becomes applicable to systems in more general forms. The manipulations with analytic and meromorphic matrices that are called for should be constructive in the sense that answers are always provided in terms of 'known' functions, i. e., functions of which we can compute the values to the desired accuracy whenever this is needed. Techniques from constructive function theory should be of help here; old-fashioned as they may seem, the advent of software for symbolic manipulation may revive these techniques as a valuable and practical tool for the dynamic modeling of complicated structures. Other research topics in this connection will include the further development of the representation theory for linear dynamical systems, with particular attention for the special properties of mechanical structures, following up on the work in [15] and [16].

\section{REFERENCES}

1. H. Blomberg, R. Ylinen (1983). Algebraic Theory for Multivariable Linear Systems, Ac. Press, London.

2. F. M. Callier, C. A. Desoer (1982). Multivariable Feedback Systems, Springer, New York.

3. G. Conte, A. M. Perdon (1986). Zeros of cascade compositions. C. I. Byrnes, A. Lindquist (eds.). Frequency Domain and State Space Methods for Linear Systems, North-Holland, Amsterdam, 23-34.

4. J. B. Conway (1978). Functions of One Complex Variable, Springer, New York.

5. M. DAviES, B. Dawson (1984). A distributed element method for vibration analysis of flexible spacecraft based on transfer matrices. (Manuscript.)

6. B. A. Francis (1987). A Course in $H_{\infty}$ Control Theory, Lect. Notes Contr. Inf. Sci., Springer, Berlin.

7. A. Hurwitz, R. Courant (1925). Funktionentheorie, Julius Springer, Berlin.

8. T. Kailath (1980). Linear Systems, Prentice-Hall, Englewood Cliffs, N. J.

9. V. KuCERA (1979). Discrete Linear Control: The Polynomial Equation Approach, Wiley, London.

10. C. C. MacDuffee (1956). The Theory of Matrices, Chelsea, New York. (Reprint of original, 1933.)

11. E. C. PESTEL, F. A. LECKIE (1963). Matrix Methods in Elastomechanics, McGraw-Hill, New York.

12. D. Poelaert (1983). DISTEL, a distributed element program for dynamic modelling and response analysis of flexible structures. 4th Symposium on Dynamics and Control of Large Structures.

13. D. POELAERT (1984). SCOLE dynamic analysis and frequency response using DISTEL software, ESTEC Working Paper 1395, ESTEC, Noordwijk.

14. H. H. Rosenbrock (1970). State Space and Multivariable Theory, Wiley, New York.

15. A. J. van DER SChaFt (1984). System Theoretic Descriptions of Physical Systems, CWI Tract 3, CWI, Amsterdam.

16. J.M.SChUMACHER (1988). State representations of linear systems with output constraints, Report OS-R88.., CWI, Amsterdam.

17. M. VIDYASAgar (1985). Control System Synthesis; A Factorization Approach, MIT Press, Cambridge, MA.

18. J.H.M. WEDDERBURN (1915). On matrices whose coefficients are functions of a single variable. Trans. Amer. Math. Soc. $16,328-332$.

19. W. H. Wittrick, F.W. WiLliams (1971). A general algorithm for computing natural frequencies of elastic structures. Quart. Journ. Mech. Appl. Math. XXIV, 263-284.

20. W. A. Wolovich (1974). Linear Multivariable Systems, Springer, New York. 